\title{
CriaçÃo de EMPRESAS: UM PROCESSO MAIS RÁPIDO E FÁCIL RESULTA EM EMPRESAS DE MELHOR DESEMPENHO?
}

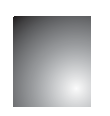

\author{
New Venture Creation: a process faster and easier results in \\ better busines performance?
}

\author{
Cândido Borges \\ Professor Adjunto na Faculdade de Administração, Contábeis e Economia da Universidade Federal de Goiás - Goiânia - Go, Brasil. \\ E-mail: candidoborges@gmail.com
}

Louis Jacques Filion

Rogers-J. A. Bombardier Chair of Entrepreneurship, HEC - Montreal - Canadá. E-mail: louisjacques.filion@hec.ca

\section{Germain Simard}

Rogers-J. A. Bombardier Chair of Entrepreneurship, HEC - Montreal - Canadá. E-mail: germain.simard@hec.ca

\section{Resumo}

As pesquisas realizadas sobre o desempenho de novas empresas se concentraram principalmente em analisar a influência das características e dos recursos dos empreendedores e das empresas sobre esse desempenho. Poucos foram os estudos que investigaram a influência do processo de criação de uma empresa sobre o seu desempenho. O objetivo deste artigo consiste em investigar se dois dos elementos do processo de criação - o tempo que os empreendedores necessitam para criar uma empresa $e$ as dificuldades que eles encontram durante a criação - influenciam o desempenho da nova empresa. A pesquisa consistiu em um survey com 175 novas empresas. Os resultados indicam que o desempenho e a rapidez com que estas atividades são realizadas são dois fatores independentes, que não exercem efeitos um sobre o outro. Sobre o grau de dificuldades encontrado, os resultados mostram que no processo de criação existem dificuldades ligadas aos recursos financeiros (acesso e gestão) e ao mercado (compreensão, avaliação e acesso) que influenciam o desempenho das novas empresas.

Palavras-chave: Empreendedorismo. Criação de Empresa. Desempenho de Novas Empresas.

\section{Abstract}

The research on the performance of new ventures has focused mainly on analyzing the influence of the characteristics and resources of entrepreneurs and their companies on this performance. Few studies have investigated the influence of the process of creating a new venture on its performance. In response to this gap, the aim of this paper is to investigate whether the two elements of the creation process - the time that entrepreneurs need to create the company and the difficulties they encounter during the creation influence the performance of the new company. This article presents the results of a survey with 175 new ventures. The results of this analysis indicate that the performance and speed with which these activities take place are two independent factors do not exert an effect on the other. On the second element - the degree of difficulties encountered - the results show that certain barriers influence the performance of new firms.

Key words: Entrepreneurship. New Venture Creation. Start-up Performance. 


\section{INTRODUÇÃo}

Uma das preocupações de um empreendedor que se prepara para criar uma empresa consiste em saber quais são os fatores que podem favorecer o sucesso $e$ o desempenho do negócio que pretende criar. Como posso criar uma empresa que não somente sobreviva, mas que tenha também um bom desempenho? - É o tipo de questão que habita a mente dos empreendedores, quando iniciam a criação de uma empresa.

$\mathrm{Na}$ literatura, a resposta a esse questionamento continua insuficiente, não obstante o fato de o desempenho de novas empresas ser um tema pelo qual os pesquisadores se interessam há muito tempo (BRUSH; VANDERWERF, 1992; COOPER, 1993; DUCHESNEAU; GARTNER, 1990). Pesquisas sobre o desempenho de novas empresas já mostraram as influências das características e dos recursos do empreendedor e da empresa (COOPER; GIMENO-GASCON; WOO, 1994; SAMMUT, 1998) ou do ambiente (ALDRICH, 1999) sobre este desempenho. Mas poucos são os estudos que se preocuparam em investigar como o processo de criação de uma empresa pode influenciar o seu desempenho.

Ora, o processo é um fator que pode também influenciar o desempenho das novas empresas. Ele forma com o ambiente, com o empreendedor e com a nova empresa as quatro dimensões principais da criação de uma empresa e, assim como as três outras dimensões, deve também ser objeto de análise para uma completa compreensão do fenômeno (FAYOLLE, 2004; FILION, 2001; GARTNER, 1985; JULIEN; MARCHESNAY, 1996; VERSTRAETE; SAPORA, 2006). Frente a essa lacuna, o objetivo deste artigo é investigar se dois dos elementos do processo de criação - o tempo que os empreendedores necessitam para criar a empresa e as dificuldades que eles encontram durante a criação influenciam o desempenho da nova empresa.

Este artigo é estruturado em cinco seções, sendo a primeira esta introdução. A segunda seção trata do processo de criação como uma dimensão que pode influenciar o desempenho das novas empresas. A terceira descreve a metodologia empregada para realizar este estudo. A quarta apresenta e analisa os resultados. Finalmente, a quinta seção conclui com sugestões às pessoas e organizações envolvidas com o campo do empreendedorismo.
Como o termo "processo" pode ter diferentes significados, neste texto nós utilizamos a definição que Borges, Simard e Filion (2005) dão para processo de criação de empresas. Segundo esses autores, este processo compreende o conjunto de atividades que o empreendedor realiza para conceber, organizar $e$ lançar uma empresa.

\section{Processo de Criação como Dimensão que Influencia o Desempenho das Novas Empresas}

Existem quatro grandes componentes do processo empreendedor que influenciam a criação de uma empresa. O primeiro é o empreendedor ou os empreendedores que criam a empresa. O segundo é a empresa que o empreendedor pretende criar. O terceiro é o ambiente onde a empresa é criada. O quarto componente é o processo de criação da nova empresa. (BRUYAT, 2001; GARTNER, 1985)

Entretanto, quando o objeto de estudo é o desempenho da nova empresa, estes quatro componentes não receberam dos pesquisadores em empreendedorismo uma atenção igual. Os estudos sobre este tema analisaram principalmente os três primeiros fatores - 0 empreendedor, a empresa e o ambiente. O quarto fator, que é o processo, continua pouco estudado. Essa é a conclusão à qual é possível chegar com o exame da literatura feito por Sammut (1998) sobre os fatores de sucesso das novas empresas. Essa autora encontrou fatores ligados ao empreendedor, ao ambiente e à organização, mas nenhum fator ligado ao processo de criação, mostrado na Tabela 1.

É necessário ressaltar que a síntese de Sammut (1998) mostrada na tabela anterior é de 1998, e que entre 1998 e 2008 foram feitos vários estudos sobre os fatores que influenciam o desempenho das novas empresas. Todavia, após uma pesquisa na literatura sobre o desempenho de novas empresas, publicada após 1998, constatou-se que o número de estudos que consideraram o fator processo continua pequeno. Esta pesquisa bibliográfica foi realizada em quatro bancos de dados: ABI/INFORM Global, Business Sources Premier, Kluwer Online e Science Direct.

Entre os autores que analisaram a relação entre o processo de criação e o desempenho da empresa cria- 
Tabela 1: Fatores de sucesso das novas empresas - síntese da literatura feita por Sammut (1998)

\begin{tabular}{|c|c|c|}
\hline $\begin{array}{c}\text { FATORES LIGADOS AO } \\
\text { EMPREENDEDOR }\end{array}$ & $\begin{array}{c}\text { FATORES LIGADOS AO } \\
\text { AMBIENTE }\end{array}$ & $\begin{array}{c}\text { FATORES LIGADOS À } \\
\text { EMPRESA }\end{array}$ \\
\hline $\begin{array}{l}\text { Parentes } \\
\text { empreendedores } \\
\text { Experiência } \\
\text { Assume riscos } \\
\text { moderadamente } \\
\text { Capacidade de } \\
\text { aprendizagem } \\
\text { Controle da situação } \\
\text { Define bem seu } \\
\text { negócio } \\
\text { Gestão eficaz do tempo }\end{array}$ & $\begin{array}{l}\text { Estudo do mercado } \\
\text { Existência e } \\
\text { utilização de } \\
\text { serviços de apoio - } \\
\text { acompanhamento } \\
\text { Obtenção de capital } \\
\text { em quantidade } \\
\text { suficiente } \\
\text { Gestão eficaz da } \\
\text { informação } \\
\text { Desenvolvimento } \\
\text { de redes - parcerias } \\
\text { Forma de entrada } \\
\text { no mercado }\end{array}$ & $\begin{array}{l}\text { Especialização } \\
\text { dos empregados } \\
\text { Delegação e } \\
\text { participação no } \\
\text { processo decisório. }\end{array}$ \\
\hline
\end{tabular}

Fonte: Sammut (1998 p. 30, Tabela 4)

da, encontram-se Chandler, Honig e Wiklund (2005), Cooper e Mehta (2003), Delmar e Shane (2002, 2003), assim como Gasse, Diochon e Menzies (2004). Estes autores analisaram diferentes aspectos do processo de criação e a influência deles sobre o desempenho das novas empresas.

Delmar e Shane (2002, 2003), por exemplo, analisaram a criação de 223 novas empresas na Suécia e concluíram que dois tipos de atividade ligadas ao processo de criação devem ser realizados no seu início, para que a empresa tenha melhor desempenho. São, em primeiro lugar, as atividades ligadas ao planejamento, como a redação do plano de negócio e a busca de informações sobre o mercado. Em segundo lugar encontram-se as atividades ligadas à legitimação, como o registro legal da empresa.

Gasse, Diochon e Menzies (2004) fizeram um estudo longitudinal sobre a criação de 119 empresas no Canadá. Os autores encontraram que as empresas que tiveram sucesso neste processo realizaram mais atividades concretas e tangíveis, como desenvolver um produto para a venda ou abrir uma conta em banco.

Um terceiro estudo, o de Cooper e Metha (2003), não encontrou nenhuma relação entre o tempo (em número de meses) que os empreendedores dedicaram à preparação da criação da empresa e o seu desempenho ( $n=2992$, Estados Unidos). Todavia, esses autores não analisaram em detalhe as atividades de prepara- ção. Eles consideraram como tempo de preparação o intervalo de meses entre o começo das despesas do empreendedor com o projeto de empresa e a realização da primeira venda.

Finalmente, Chandler, Honig e Wiklund (2005) analisaram a relação entre mudança na equipe empreendedora e o desempenho das novas empresas com base em duas amostras. A primeira na Suécia $(n=408)$, a segunda nos Estados Unidos $(n=124)$. Esses autores concluíram que as empresas que começam com uma equipe empreendedora suficientemente grande (sem, entretanto indicar um tamanho específico) para poderem perder membros durante o processo de criação têm um desempenho melhor que aquelas que precisam adicionar sócios durante este processo.

Apesar de seu pequeno número, os quatro estudos relatados possibilitam constatar que o processo de criação pode, efetivamente, influenciar o desempenho das novas empresas. Essa influência é estuda de forma mais profunda na pesquisa apresentada neste artigo. A metodologia utilizada para realizar esta pesquisa é apresentada na próxima seção.

\section{Metodologia}

Nesta seção será, inicialmente, apresentada a amostra de empresas que participaram da pesquisa e, posteriormente, serão apresentados os procedimentos de coleta de dados. Em seguida, as variáveis de análise serão definidas. Finalmente, na terceira subseção, será descrita a forma utilizada para tratar e analisar os dados.

\subsection{Amostra e Coleta de Dados}

Os dados analisados neste artigo foram coletados como parte de um projeto de pesquisa sobre a criação de empresas. Esta pesquisa foi realizada com 201 empresas que possuíam, no momento da pesquisa, entre dois e quatro anos e empregavam no mínimo quatro pessoas. Para constituir a amostra, foi solicitada a colaboração de organizações governamentais e paragovernamentais, que trabalham no apoio aos criadores de empresas. Todas as empresas da amostra foram indicadas por essas organizações. Como, entre as 201 empresas da amostra, 26 tinham dados faltantes, 
apenas 175 casos foram retidos na amostra analisada neste artigo. Essas empresas são de todos os setores e regiões da Província do Québec, Canadá.

Dois questionários contendo questões majoritariamente fechadas foram utilizados como instrumento de coleta dos dados: questionário "A" e questionário "B". O questionário "A" abordava as informações sobre o empreendedor e a nova empresa, como o número de empregados da empresa, sua localização, seu faturamento, a formação e as experiências do empreendedor, bem como as fontes de financiamento da empresa. $\mathrm{O}$ questionário "B" trazia questões referentes ao processo de criação da empresa e foi estruturado seguindo o modelo de criação de empresas de Borges, Simard, e Filion (2005). Esse modelo divide o processo de criação em quatro etapas: 1) iniciação, 2) preparação, 3) lançamento e 4) consolidação.

Os questionários foram pré-testados em duas fases. Na primeira, eles foram apresentados a cinco profissionais que trabalham em organizações de apoio à criação de empresas. Na segunda etapa, ele foi aplicado a empreendedores cujas empresas respondiam aos critérios da pesquisa. Após cada uma das fases do pré-teste, a equipe de pesquisa reavaliava as questões dos questionários e a experiência de utilizá-los, realizando, em seguida, as correções julgadas necessárias.

A coleta dos dados foi feita entre junho de 2004 e março de 2005, em duas fases. Em primeiro lugar, o empreendedor respondia ao questionário "A", que lhe foi enviado, segundo sua preferência, por correio eletrônico, por fax ou por correio regular. Em seguida, um membro da equipe de pesquisadores tinha um encontro com o empreendedor, em que ele revisava o questionário "A", respondido anteriormente pelo empreendedor, $e$, na sequência, aplicava o questionário "B". A duração média destes encontros foi de 90 minutos.

\subsection{Variáveis de Análise}

Nesta subseção serão apresentadas as variáveis utilizadas no estudo relatado neste artigo. A variável dependente é o desempenho. As variáveis independentes são dois elementos do processo de criação de uma empresa: 1) o tempo e 2) as dificuldades.

\subsubsection{Desempenho Como Variável Dependente}

A variável dependente deste estudo é o desempenho. Mas o que significa "desempenho de uma nova empresa"? Como medir esse desempenho? Chandler e Hanks (1993) fizeram um exame da literatura sobre o desempenho de novas empresas e constataram que, quando usados dados fornecidos pelos empreendedores, as três formas mais utilizadas para medir esse desempenho são: 1) categorias objetivas (crescimento e faturamento); 2) medidas subjetivas de satisfação do empreendedor com o desempenho da empresa; 3) medidas subjetivas de comparação com competidores. Os pesquisadores investigaram a pertinência, a disponibilidade, a confiança e a validade dessas três categorias em uma amostra de 120 novas empresas nos Estados Unidos. O resultado dessa investigação foi que as medidas mais consistentes são, na ordem: as categorias objetivas, as medidas subjetivas de comparação com os competidores e as medidas subjetivas de satisfação do empreendedor com o desempenho.

A Tabela 2 mostra como certos estudos sobre o desempenho de novas empresas, realizados depois de 2000, mediram o desempenho. Nela é possível constatar que as medidas indicadas por Chandler e Hanks (1993) continuam a ser predominantes.

Tabela 2: Exemplos de medida do desempenho de novas empresas

\begin{tabular}{|c|c|}
\hline Autores & INDICADORES UTILIZADOS \\
\hline $\begin{array}{l}\text { Chandler, Honig e } \\
\text { Wiklund (2005) }\end{array}$ & $\begin{array}{l}\text { Alcance do ponto de equilíbrio } \\
\text { e crescimento do faturamento }\end{array}$ \\
\hline $\begin{array}{l}\text { Chrisman, McMullan } \\
\text { e Hall (2005) }\end{array}$ & $\begin{array}{l}\text { Tamanho (número de } \\
\text { funcionário e faturamento). }\end{array}$ \\
\hline Cooper e Mehta (2003) & $\begin{array}{l}\text { Continuar a existir (sobreviver), } \\
\text { o empreendedor tem renda } \\
\text { com a empresa, crescimento } \\
\text { do número de funcionários. }\end{array}$ \\
\hline Wiklund e Shepherd (2005) & $\begin{array}{l}\text { Crescimento do número de } \\
\text { funcionários e do faturamento. E a } \\
\text { avaliação que os empreendedores } \\
\text { fazem do desempenho da empresa } \\
\text { em comparação a seus competidores. }\end{array}$ \\
\hline Perreault et al. (2007) & $\begin{array}{l}\text { Tamanho (número de } \\
\text { funcionário), longevidade (idade } \\
\text { da empresa) e faturamento. }\end{array}$ \\
\hline
\end{tabular}




\begin{tabular}{|ll|} 
Reid e Smith (2000) & $\begin{array}{l}\text { Crescimento do número de } \\
\text { funcionários, retorno sobre o } \\
\text { investimento e produtividade. }\end{array}$ \\
Watson (2007) & $\begin{array}{l}\text { Continuar a existir (sobreviver), } \\
\text { crescimento do faturamento, } \\
\text { retorno sobre o investimento. }\end{array}$ \\
\hline
\end{tabular}

Fonte: Elaborado pelos autores deste artigo

Para construir um indicador de desempenho mais completo, este estudo optou por considerar medidas objetivas e medidas subjetivas. Um Índice de Desempenho (ID) foi, então, determinado com base no faturamento da empresa (FE), no fato de a empresa ter alcançado o ponto de equilíbrio $(\mathrm{Q})$ e na autoavaliação que empreendedor faz do desempenho de sua empresa em relação a seus competidores (AE). As duas primeiras constituem-se em medidas objetivas e a terceira em medida subjetiva.

O Índice de Desempenho (ID) foi calculado segundo a equação seguinte: $\mathrm{ID}=(\mathrm{FE} \times \mathrm{Q} \times \mathrm{AE}) . \mathrm{O}$ faturamento (FE) pode ter um valor de 1 a 7 (questão 20 do questionário $\mathrm{A}$ ): valor 1 para um faturamento menor do que $100000 \$$ (todos os valores em dólar canadense), valor 2 para $100001 \$$ a $250000 \$$, valor 3 para $250001 \$$ a $500000 \$$, valor 4 para $500001 \$$ a $1000000 \$$, valor 5 para $1000001 \$$ a 2,5 Milhões $\$$, valor 6 para $2500001 \$$ à 10 Milhões $\$$ e valor 7 para mais de 10 Milhões $\$$. A rentabilidade pode ter o valor 1 ou 2 (questão 35 do questionário B), onde: valor 1 para não ter alcançado o ponto de equilíbrio e 2 para ter alcançado.

A autoavaliação pode ter o valor de 1 a 5 . Este valor é originário de uma escala de 1 a 5 (1 - inferior; 2 - um pouco inferior; 3 - igual; 4 - um pouco superior; 5 - superior; NA - não se aplica), que os empreendedores utilizaram para responder a questão seguinte: "Como você situa o sucesso de sua empresa em relação ao de seus concorrentes diretos?" (questão 38 , item $\mathrm{F}$, do questionário $\mathrm{B}$ ). Os respondentes que responderam NA não são considerados nesta análise.

O ID pode ter valores que variam de 1 a 70 . Além dos casos rejeitados (previamente determinados no banco de dados), um dado faltante em qualquer uma das questões ligadas ao cálculo do índice resulta em dado faltante no ID.

\subsubsection{Tempo e Dificuldades Como Variáveis Independentes}

Dois elementos do processo de criação de empresas são usados para analisar a influência deste processo no desempenho das novas empresas. O primeiro é o tempo que os empreendedores usam para realizar as atividades necessárias à criação de uma empresa. $\mathrm{O}$ segundo são as dificuldade que eles encontram durante o processo de criação. Esses dois elementos foram escolhidos em razão de sua importância no estudo de um processo empreendedor (BIRD; WEST, 1997; LERNER; ZAHRA; KOHAVI, 2007; VAN DE VEN; POOLE, 1995) e também por comodidade, pois tratam-se de dados disponíveis e confiáveis, provenientes da pesquisa sobre criação de empresas e recolhidos diretamente junto aos empreendedores, como descrito na seção 3.1 (amostra e coleta de dados).

Em relação ao tempo, seguindo o procedimento usado por Carter, Gartner e Reynolds (1996), foi calculado o tempo em meses que os empreendedores necessitaram para realizar algumas das atividades do processo de criação. As atividades analisadas foram as seguintes: a identificação de uma oportunidade de negócios, a decisão de criar a empresa, o início do trabalho do empreendedor em tempo integral na empresa, o término do desenvolvimento do primeiro produto, a contratação do primeiro funcionário e a concretização da primeira venda. (CARTER; GARTNER; REYNOLDS, 1996; REYNOLDS; MILLER, 1992; VÉSPER, 1990)

No questionário, os empreendedores indicaram a data (mês e ano) em que eles completaram cada uma das atividades citadas no parágrafo anterior. Com esses dados, foi possível calcular o intervalo de tempo entre a decisão de criar a empresa e efetuar as outras atividades, ou seja, quanto tempo os empreendedores levaram, após a decisão de criar a empresa, para realizar cada uma das atividades.

Em relação às dificuldades, no questionário os empreendedores escolhiam, em uma escala de 1 (muito difícil) a 5 (muito fácil), o grau de dificuldade que eles encontraram para realizar as atividades que constituem as quatro etapas do processo de criação de empresas. Vários aspectos de cada uma dessas quatro etapas foram analisados e serão apresentados na sequência, 
no momento da apresentação dos resultados sobre a influência das dificuldades no desempenho.

\subsection{Tratamento dos Dados}

O software SPSS numeração foi utilizado no tratamento e na análise dos dados. Inicialmente foi realizada uma análise comparativa com o objetivo de explorar a possível influência dos diferentes fatores sobre o desempenho das novas empresas. Em segui$\mathrm{da}$, foram calculadas as correlações entre o Índice de Desempenho (ID) e esses fatores.

Como última estratégia de análise, modelos de regressão múltipla foram construídos e analisados. Os resultados dessas diferentes análises são apresentados na próxima seção deste artigo.

\section{Apresentação e Análise dos Resultados}

O Índice de Desempenho (ID) - médio das 175 novas empresas é 24,33 e a mediana é 24 . Entre essas empresas, $5 \%$ têm um Índice de Desempenho de 4 ou menos, $25 \%$ de 12 ou menos, $25 \%$ de 32 ou mais e $5 \%$ têm um ID de 50 ou mais.

\subsection{O Tempo e o Desempenho}

Foi analisada a correlação entre o Índice de Desempenho e o tempo necessário para a realização das atividades do processo de criação. Em nenhuma das atividades, uma correlação entre o Índice de Desempenho (variável dependente) e a quantidade de meses, que foram necessários para realizar as atividades em questão (variável independente), foi encontrada (veja Tabela 3). Esses resultados indicam que não existe uma correlação significativa entre o desempenho e o tempo que os empreendedores gastam para realizar as atividades necessárias à criação de uma empresa.

Além da análise da correlação entre o Índice de Desempenho e cada uma das variáveis temporais, mostradas na Tabela 3, foi construído um modelo de regressão em que o Índice de Desempenho é dependente do conjunto das variáveis temporais. Esse modelo não gerou resultados conclusivos. O coeficiente de determinação do modelo $(\mathrm{R} 2=, 061)$ mostrou-se muito fraco
Tabela 3: Correlação entre o Índice de Desempenho e o tempo necessário para realizar as atividades do processo de criação

\begin{tabular}{|c|c|c|c|}
\hline VARIÁVEL & N. & $\begin{array}{l}\text { Índice de } \\
\text { CORRELAÇão } \\
\text { DE PEARSon }\end{array}$ & $\begin{array}{c}\text { SIG. } \\
\text { (2-TAILED) }\end{array}$ \\
\hline $\begin{array}{l}\text { TPN (tempo entre a data de } \\
\text { decisão de criar a empresa e } \\
\text { a data do término da redação } \\
\text { do plano de negócios) }\end{array}$ & 158 & ,066 & ,407 \\
\hline $\begin{array}{l}\text { TRJ (tempo entre a data da } \\
\text { decisão de criar a empresa e data } \\
\text { da constituição legal da empresa) }\end{array}$ & 168 & ,040 & ,609 \\
\hline $\begin{array}{l}\text { TTI (tempo entre a data da } \\
\text { decisão de criar a empresa } \\
\text { e o início do trabalho do } \\
\text { empreendedor em tempo } \\
\text { integral na empresa) }\end{array}$ & 162 & ,031 & ,698 \\
\hline $\begin{array}{l}\text { TDP (tempo entre a data da } \\
\text { decisão de criar a empresa } \\
\text { e a data de o término } \\
\text { do desenvolvimento do } \\
\text { primeiro produto) }\end{array}$ & 165 &,- 046 &, 558 \\
\hline $\begin{array}{l}\text { TCF (tempo entre a data da } \\
\text { decisão de criar a empresa } \\
\text { e a data da contratação do } \\
\text { primeiro funcionário) }\end{array}$ & 170 &,- 001 & ,993 \\
\hline $\begin{array}{l}\text { TPV (tempo entre a data da } \\
\text { decisão de criar a empresa } \\
\text { e a data de realização } \\
\text { da primeira venda) }\end{array}$ & 169 &,- 042 & ,589 \\
\hline
\end{tabular}

Fonte: Elaborado pelos autores deste artigo

e o modelo não foi significativo. Um coeficiente de determinação de 0,061 significa que o modelo explica $6,1 \%$ da variação da variável dependente.

O resultado do modelo de regressão era previsível, já que não foi possível observar nenhuma correlação significativa entre as variáveis independentes deste modelo e o Índice de Desempenho. Mais uma vez esses resultados levam à conclusão de que o desempenho das novas empresas e a rapidez com que o empreendedor realizou as atividades do processo de criação são dois fatores independentes, que não ocasionaram efeitos significativos um sobre o outro.

Esses resultados contradizem outros estudos que mostraram que a rapidez com a qual os empreendedores realizam as atividades do processo de criação (CARTER; GARTNER; REYNOLDS, 1996) ou tomam decisões (BOURGEOIS; EISENHARDT, 1988) é um 
fator de sucesso das novas empresas. Essas diferenças entre os resultados podem ser ocasionadas por vários fatores, entre eles o tipo de amostra e o método de análise. Por exemplo, o estudo apresentado neste artigo analisou novas empresas em fase de consolidação; já Bourgeois e Eisenhardt (1988) investigaram as decisões tomadas no momento em que a empresa já estava consolidada. Além disso, como neste artigo foram analisadas 175 empresas de diferentes setores, a amostra de Bourgeois e Eisenhardt (1988) referia-se a um setor bem especifico ( $n=4$, empresas de informática, dos Estados Unidos).

\subsection{As Dificuldades e o Desempenho}

Correlações foram calculadas entre o grau de dificuldade que os empreendedores encontraram para realizar as atividades que constituem as quatro etapas do processo de criação de uma empresa (iniciação, preparação, lançamento e consolidação) e o Índice de Desempenho (ID). Os resultados referentes a cada uma dessas etapas serão apresentados nesta subseção.

\subsubsection{Dificuldades na Etapa de Iniciação}

A Tabela 4 mostra correlação entre o grau de dificuldade que os empreendedores encontraram durante a etapa de iniciação e o Índice de Desempenho (ID). Em três dos itens considerados, existe uma correlação significativa de $95 \%$ entre o Índice de Desempenho e o grau de dificuldade encontrado. Esses três itens são: Avaliar o potencial do projeto de negócio, encontrar informações sobre o produto ou serviço e informar-se sobre o setor de atividades.

Sendo esses três coeficientes de correlação positivos, é possível concluir que mais é percebido como fácil a avaliação do potencial do projeto de negócios, a localização de informações sobre o produto ou serviço e o acesso a informações sobre o setor de atividades, mais elevado será o Índice de Desempenho.

\subsubsection{Dificuldades na Etapa de Preparação}

A Tabela 5 mostra a correlação entre o grau de dificuldade que os empreendedores encontram na eta-
Tabela 4: Correlação entre o Índice de Desempenho e as dificuldades encontradas na etapa de iniciação

\begin{tabular}{|c|c|c|}
\hline Dificuldade EM RELAÇão A & $\mathbf{N}$ & $\begin{array}{c}\text { ÍnDICE DE } \\
\text { CORRELAÇão DE } \\
\text { PEARSON }\end{array}$ \\
\hline $\begin{array}{l}\text { Encontrar boas oportunidades } \\
\text { de negócio }\end{array}$ & 170 & 077 \\
\hline $\begin{array}{l}\text { Avaliar o potencial do } \\
\text { projeto de negócio }\end{array}$ & 175 &, $180(*)$ \\
\hline $\begin{array}{l}\text { Encontrar informações sobre } \\
\text { o produto ou serviço }\end{array}$ & 174 &, $165(*)$ \\
\hline Encontrar pessoas de apoio & 172 & 001 \\
\hline $\begin{array}{l}\text { Conhecer minhas capacidades } \\
\text { empreendedoras }\end{array}$ & 171 & ,133 \\
\hline $\begin{array}{l}\text { Avaliar minha capacidade de } \\
\text { desenvolver o negócio }\end{array}$ & 173 & 051 \\
\hline $\begin{array}{l}\text { Informar-me sobre o } \\
\text { setor de atividades }\end{array}$ & 174 &, $177(*)$ \\
\hline $\begin{array}{l}\text { Compreender a constituição } \\
\text { legal de uma empresa }\end{array}$ & 168 & 088 \\
\hline Conhecer as fontes de financiamento & 175 &, 144 \\
\hline Correr riscos & 175 &, 047 \\
\hline $\begin{array}{l}\text { Convencer as pessoas } \\
\text { próximas de meu projeto }\end{array}$ & 165 &,- 005 \\
\hline
\end{tabular}

Fonte: Elaborado pelos autores deste artigo

pa de preparação e o Índice de Desempenho (ID). Em seis itens foi encontrada uma correlação significativa de $99 \%$ entre o Índice de Desempenho e o grau de dificuldade. Esses itens são: compreender o mercado, elaborar o plano de marketing, manter a motivação para o projeto, encontrar tempo para fazer tudo que é necessário e negociar o financiamento. É também possível constatar, na Tabela 5, uma correlação significativa de 95\% entre o Índice de Desempenho (ID) e o grau de dificuldade em definir o produto ou serviço.

Uma vez mais, sendo sete coeficientes de correlação positivos (seis de $99 \%$ e um de $95 \%$ ), é possível concluir que quanto mais os sete itens indicados no parágrafo precedente forem percebidos como fáceis de realizar, mais o Índice de Desempenho será elevado. 
Tabela 5: Correlação entre o Índice de Desempenho e as dificuldades encontradas na etapa de preparação

\begin{tabular}{|c|c|c|}
\hline DificuldAdE EM RELAÇÃO A & $\mathbf{N}$ & $\begin{array}{c}\text { Í́NDICE DE } \\
\text { CORRELAÇão DE } \\
\text { PEARSON }\end{array}$ \\
\hline Compreender o mercado & 175 &, $249(* *)$ \\
\hline Definir o produto/serviço & 175 &, $187(*)$ \\
\hline Elaborar o plano de marketing & 158 &, $211(* *)$ \\
\hline Elaborar o plano financeiro & 170 & ,090 \\
\hline Conceber o sistema organizacional & 171 & ,044 \\
\hline Elaborar o plano de recursos humanos & 155 &,- 081 \\
\hline Manter a motivação para o projeto & 175 & $213(* *)$ \\
\hline $\begin{array}{l}\text { Encontrar tempo para fazer } \\
\text { tudo que é necessário }\end{array}$ & 175 &, $214(* *)$ \\
\hline Discutir com os contatos de apoio & 162 & ,076 \\
\hline Negociar o financiamento & 165 &, $221(* *)$ \\
\hline Estabelecer um sistema contábil & 168 &, 112 \\
\hline Encontrar os parceiros financeiros & 153 &, $237(* *)$ \\
\hline Encontrar sócios & 125 &, 109 \\
\hline
\end{tabular}

* = correlação significativa de $95 \%$; ** = correlação significativa de $99 \%$.

Fonte: Elaborado pelos autores deste artigo

\subsubsection{Dificuldades na Etapa de Lançamento}

A Tabela 6 mostra a correlação entre o grau de dificuldade que os empreendedores encontram durante a etapa de lançamento e o Índice de Desempenho (ID). Em quatro itens existe uma correlação significativa de 99\% entre o Índice de Desempenho e o grau de dificuldade encontrado em relação a estes itens. Esses quatro itens são: manter a motivação, lidar com a quantidade insuficiente de recursos disponíveis, lidar com o excesso de custos e negociar com o banco. Além disso, no item "adquirir os conhecimentos para conduzir bem o projeto" existe uma correlação positiva de $95 \%$ entre o Índice de Desempenho e este elemento.
Tabela 6: Correlação entre o Índice de Desempenho e as dificuldades encontradas na etapa de lançamento

\begin{tabular}{|c|c|c|}
\hline DificuldAdE RELACIONADA & $\mathbf{N}$ & $\begin{array}{c}\text { ÍNDICE DE CORRELA- } \\
\text { ÇÃo de PEARSON }\end{array}$ \\
\hline $\begin{array}{l}\text { A adquirir os conhecimentos } \\
\text { para conduzir bem o projeto }\end{array}$ & 175 &, $152(*)$ \\
\hline A contratar funcionários & 172 &, 015 \\
\hline A ter acesso às tecnologias & 147 & ,026 \\
\hline $\begin{array}{l}\text { A registrar legalmente a empresa } \\
\text { e preencher os formulários legais }\end{array}$ & 172 & ,087 \\
\hline A manter a motivação & 175 &, $218(* *)$ \\
\hline $\begin{array}{l}\text { A conciliar o tempo com } \\
\text { as outras atividades }\end{array}$ & 174 &, 074 \\
\hline $\begin{array}{l}\text { À quantidade insuficiente } \\
\text { de recursos disponíveis }\end{array}$ & 146 &, $260(* *)$ \\
\hline à relação entre os sócios & 138 & ,009 \\
\hline Ao acesso e a venda aos clientes & 170 &, 027 \\
\hline $\begin{array}{l}\text { Ao desenvolvimento do } \\
\text { primeiro produto ou serviço }\end{array}$ & 169 &, 149 \\
\hline Ao acesso aos fornecedores & 165 & ,099 \\
\hline $\begin{array}{l}\text { À organização das instalações } \\
\text { e equipamentos }\end{array}$ & 171 &, 150 \\
\hline Ao excesso de custos & 142 &, $292(* *)$ \\
\hline A Negociar com o banco & 157 &, $267(* *)$ \\
\hline A gerir o pessoal & 166 &,- 006 \\
\hline
\end{tabular}

Fonte: Elaborado pelos autores deste artigo

\subsubsection{Dificuldades na Etapa de Consolidação}

A Tabela 7 mostra a correlação entre o grau de dificuldade que os empreendedores encontram durante a etapa de consolidação e o Índice de Desempenho (ID). Em três dos itens apresentados existe uma correlação significativa de $99 \%$ entre o Índice de Desempenho e o grau de dificuldade encontrado. Estes itens são os seguintes: obter o financiamento para manter ou crescer as operações, alcançar o ponto de equilíbrio e lidar com o excesso de custos. Também é possível constatar na Tabela 7 uma correlação significativa 
de $95 \%$ entre o Índice de Desempenho e o grau de dificuldade encontrado nos seguintes itens: vender o primeiro produto ou serviço, manter a motivação, o acesso aos fornecedores, planejar as operações e adquirir os conhecimentos para conduzir a empresa.

Tabela 7: Correlação entre o Índice de Desempenho e as dificuldades encontradas na etapa de consolidação

\begin{tabular}{|c|c|c|}
\hline DiFICULDADE RELACIONADA & N. & $\begin{array}{c}\text { ÍNDICE DE } \\
\text { CORRELAÇão } \\
\text { DE PEARSON }\end{array}$ \\
\hline $\begin{array}{l}\text { A obter o financiamento para } \\
\text { manter ou crescer as operações }\end{array}$ & 157 &, $313(* *)$ \\
\hline $\begin{array}{l}\text { A contratação e a gestão de } \\
\text { recursos humanos }\end{array}$ & 174 &,- 001 \\
\hline A alcançar o ponto de equilibrio & 174 &, $257(* *)$ \\
\hline A vender o primeiro produto ou serviço & 172 &, $182(*)$ \\
\hline $\begin{array}{l}\text { A realizar as atividades de } \\
\text { venda ou marketing }\end{array}$ & 157 & ,079 \\
\hline Ao acesso às tecnologias & 151 & ,053 \\
\hline A manter a motivação & 174 &, $170(*)$ \\
\hline À relação entre os sócios & 139 &,- 016 \\
\hline À relação com os parceiros financeiros & 159 &, 140 \\
\hline Ao acesso aos clientes & 175 & ,079 \\
\hline Ao acesso aos fornecedores & 166 &, $184(*)$ \\
\hline A planejar as operações & 173 &, $151\left(^{*}\right)$ \\
\hline A conciliar o tempo com as outras atividades & 175 &, 134 \\
\hline Ao excesso de custos & 150 &, $230(* *)$ \\
\hline À concorrência & 172 &, 028 \\
\hline $\begin{array}{l}\text { A adquirir os conhecimentos } \\
\text { para conduzir a empresa }\end{array}$ & 172 &, $160(*)$ \\
\hline
\end{tabular}

* = correlação significativa de $95 \%$; ** = correlação significativa de $99 \%$.

Fonte: Elaborado pelos autores deste artigo

Sendo positiva a correlação dos itens indicados no parágrafo precedente, é possível concluir que quanto mais esses itens forem fáceis para o empreendedor, maior será o Índice de Desempenho.

\subsubsection{Consolidação de Resultados Sobre a Relação Entre Dificuldades e Desempenho}

Analisando os resultados das dificuldades encontradas nas quatro etapas do processo de criação, é possível constatar que existem dificuldades que influenciam o desempenho das novas empresas. Organizando, por categoria, essas dificuldades que influenciam o Índice de Desempenho (veja Tabela 8), percebe-se que elas são, principalmente, de dois tipos: dificuldades ligadas aos recursos financeiros (acesso $e$ gestão) e dificuldades ligadas ao mercado (compreensão, avaliação e acesso). O aspecto motivação também aparece como elemento importante, assim como a aquisição de conhecimentos.

Tabela 8: Dificuldades que tem uma correlação significativa com o desempenho, por categoria

\begin{tabular}{|ll}
\hline CATEGORIA & \multicolumn{1}{c}{ DificuldadE EM RELAção A } \\
& Negociar o financiamento (Preparação) \\
& Encontrar os parceiros financeiros (Preparação) \\
& A quantidade insuficiente de recursos \\
disponíveis (Lançamento)
\end{tabular}

Fonte: Elaborado pelos autores deste artigo 


\section{Conclusão e Recomendações}

O objetivo deste artigo é o de contribuir ao melhor conhecimento da influência do processo de criação de uma nova empresa sobre o seu desempenho. Para alcançar este objetivo, a influência de dois elementos do processo de criação - o tempo que os empreendedores necessitam para criar a empresa $e$ as dificuldades que eles encontram durante a criação - sobre o desempenho da nova empresa foi analisada.

Os resultados dessa análise não mostraram correlação significativa entre o primeiro elemento (tempo) e o desempenho. Já, na relação entre o segundo elemento (grau das dificuldades) e o desempenho das novas empresas, a correlação é significativa.

No elemento tempo, o aspecto estudado foi a rapidez com que os empreendedores realizam as atividades do processo de criação. Os resultados deste estudo indicam que o desempenho e a rapidez com que essas atividades são realizadas são dois fatores independentes, que não exercem efeitos um sobre o outro. Considerando que se vive em uma época onde a mensagem que é predominante vinculada é a de que tudo deve ser feito rapidamente, esses resultados são importantes, pois mostram que "rapidez" não significa necessariamente um melhor desempenho. Com base nessa conclusão, recomenda-se aos empreendedores $e$ as pessoas que trabalham no apoio ao empreendedorismo, que uma excelente preparação (por exemplo, estudando previamente o mercado) à criação da empresa não pode ser negligenciada em razão do tempo que tal preparação pode requerer.

Sobre o segundo elemento - o grau de dificuldades encontrado -, os resultados mostram que nas quatro etapas do processo de criação existem dificuldades que podem influenciar o desempenho das novas empresas. Reagrupando por categorias as dificuldades que influenciam o desempenho, foi possível constatar que elas são principalmente ligadas aos recursos financeiros (acesso e gestão) e ao mercado (compreensão, avaliação e acesso). Nesse sentido, seria importante que os acadêmicos e as pessoas que apoiam os empreendedores, consagrassem mais esforços ao desenvolvimento $e$ à oferta de ferramentas $e$ serviços com potencial de ajudar os empreendedores a melhor gerir as atividades ligadas aos recursos financeiros e ao mercado.
É importante que outras pesquisas sobre o fator tempo sejam realizadas. Neste artigo apenas o tempo usado para realizar as atividades do processo de criação foi objeto de análise. Entretanto, o fator tempo contém outras dimensões (BIRD; WEST, 1997). Outras pesquisas poderiam, por exemplo, estudar mais a gestão do tempo durante o processo de criação - fator que apareceu como uma das dificuldades dos empreendedores (veja seção 4.2). Ou ainda, tratar da relação do tempo com apenas uma das atividades do processo de criação, como Filion et al. (2007) sugeriram em relação à identificação da oportunidade de negócio.

\section{REFERÊNCIAS}

ALDRICH, H. Organizations evolving. London: Sage Publications, 1999.

BIRD, B.; WEST, G. P. Time and entrepreneurship. Entrepreneurship: Theory and Practice, Malden, Estados Unidos, v. 22, n. 2, p. 5-9, 1997.

\section{BORGES, C.; SIMARD, G.; FILION, L. J. Création}

d'entreprises - Examen de la documentation, Cahier de recherche 2005-02, Chaire d'entrepreneuriat Rogers-J.A.Bombardier, HEC Montréal, 2005.

BOURGEOIS, L. J.; EISENHARDT, K. Strategic decision process in high velocity environment: four cases in the microcomputer industry. Management Science, Hanover, Estados Unidos, v. 34, n. 7, p. 816-835, 1988.

BRUSH, C.; VANDERWERF, P. A comparison of methods and sources for obtaining estimates of new venture performance. Journal of Business Venturing, Bloomington, Estados Unidos, v. 7, n. 2, p. 157-170, 1992.

BRUYAT, C. Créer ou ne pas créer? Une modélisation du processus d'engagement dans un projet de création d'entreprise. Revue de l'entrepreneuriat, Bruxelas, Bélgica, v. 1, n. 1, p. 25-42, 2001.

CARTER, N. M.; GARTNER, W. B.; REYNOLDS, P. D. Exploring start-up event sequences. Journal of Business Venturing, Bloomington, Estados Unidos, v. 11, n. 3, p. 151-166, 1996. 
CHANDLER, G.; HONIG, B.; WIKLUND, J. Antecedents, moderators, and performance consequences of membership change in new venture teams. Journal of Business Venturing, Bloomington, Estados Unidos, v. 20, n. 5, p. 705-725, 2005.

CHANDLER, G.; HANKS, S. Measuring the performance of emerging businesses: a validation study. Journal of Business Venturing, Bloomington, Estados Unidos, v. 8, n. 5, p. 391-408, 1993.

CHRISMAN, J.; McMULLAN, E.; HALL, J. The influence of guided preparation on the long-term performance of new ventures. Journal of Business Venturing, Bloomington, Estados Unidos, v. 20, n. 6, p. 769-791, 2005.

COOPER, A. C. Challenges in predicting new firm performance. Journal of Business Venturing, Bloomington, Estados Unidos, v. 8, n. 3, p. 241-253, 1993.

COOPER, A.; GIMENO-GASCON, J.; WOO, C. Initial human and financial capital as predictors of new venture performance. Journal of Business Venturing, Bloomington, Estados Unidos, v. 9, n. 5, p. 371-395, 1994.

COOPER, A.; MEHTA, S. Preparation for entrepreneurship - Does it matter? In: ANNUAL ENTREPRENEURSHIP RESEARCH CONFERENCE, 23, 2003, Anais... Babson College, 2003.

DUCHESNEAU, D.; GARTNER, W. A profile of new venture success and failure in an emerging industry. Journal of Business Venturing, Bloomington, Estados Unidos, v. 5, n. 5, p. 297-312, 1990.

DELMAR, F; SHANE, S. What firm founders do: a longitudinal study of the start-up process. In: BYGRAVE, W. D. et al. (Ed..), Frontiers of Entrepreneurship Research. Wellesley: Babson College, 2002. p. 632-645.

Does business planning facilitate the development of new ventures? Strategic Management Journal, Malden, Estados Unidos, v. 24, n. 12, p. 1.165-1.185, 2003.
FAYOLLE, A. Compréhension mutuelle entre les créateurs d'entreprise et les accompagnateurs: une recherche exploratoire sur des différences de perception. Management International, Montreal, Canadá, v. 8, n. 2, p. 1-14, 2004.

FAYOLLE, A.; FILION, L. J. Devenir entrepreneur: des enjeux aux outils. Paris: Village Mondial, 2006.

FILION, L. J. (Ed.) Réaliser son projet d'entreprise. 3. ed. Montréal: Éditions Transcontinental, 2001.

FILION, L. J.; BORGES, C.; SIMARD, G. Étude du processus de création d'entreprises structuré en quatre étapes. Comunicação apresentada no CONGRÈS INTERNATIONAL FRANCOPHONE SUR LA PME, 8, 2006. Anais... Fribourg. Publiée dans les Actes, 2006.

FILION, L. J. et al. Lidentification d'une occasion d'affaires en contexte d'entrepreneuriat ethnique. Revue Internationale P.M.E. Quebec, Canadá, v. 20, n. 2, p. 33-57, 2007.

GARTNER, W. A conceptual framework for describing the phenomenon of new venture creation. Academy of Management Review, Briarcliff Manor, Estados Unidos, v. 10 , n. 4 , p. 696-706, 1985.

GASSE, Y.; DIOCHON, M.; MENZIES, T. V. Les entrepreneurs émergents dont l'entreprise est devenue opérationnelle et les autres : comparaisons lors de la conception du projet d'entreprise. Journal of Small

Business \& Entrepreneurship, Saskatchewan, Canadá, v. 17, n. 2, p. 117-134, 2004.

JULIEN, P. A.; MARCHESNAY, M. L'entrepreneuriat. Paris: Economica, 1996.

LERNER, M.; ZAHRA, S.; KOHAVI, Y. Time and corporate entrepreneurship. In: LUMPKIN, G. T.; KATZ, J. (Ed.) Entrepreneurial Strategic Processes, London: Elsevier/JAI, 2007. p. 187-221.

PERREAULT, C. et al. Social capital and business performance: ethnic enterprises in Canada.

International Journal of Business and Globalisation, Genebra, Suiça, v. 1, n. 2, p. 145-160, 2007. 
REID, G.; SMITH, J. What makes a new business start-up successful? Small Business Economics, Nova Iorque, Estados Unidos, v. 14, n. 3, p. 165-82, 1992.

REYNOLDS, P.; MILLER, B. New firm gestation: conception, birth, and implications for research. Journal of Business Venturing, Bloomington, Estados Unidos, v. 7, n. 5, p. 405-417, 1992.

SAMMUT, S. Jeune entreprise: la phase cruciale du démarrage. Paris: L'Harmattan, 1992.

VAN DE VEN, A. H.; POOLE, M. S. Explaining development and change in organizations. Academy of Management Review, Briarcliff Manor, Estados Unidos v. 20, n. 3, p. 510-540, 1995.

VERSTRAETE, T.; SAPORTA, B. Création d'entreprise et entrepreneuriat. Editions de l'ADREG. 2006.

Disponível em: < http://www.editions-adreg.net>. Acesso em: 12/05/2007

VESPER, K. H. New Venture Strategies. Englewood Cliffs: Prentice Hall, 2007.

WATSON, J. (2007). Modeling the relationship between networking and firm performance. Journal of Business Venturing, Bloomington, Estados Unidos, v. 22, n.6, p.852-874, 1990.

WIKLUND, J.; SHEPHERD, D. Entrepreneurial orientation and small business performance: a configurational approach. Journal of Business Venturing, Bloomington, Estados Unidos, v. 20, n. 1, p. 71-91, 2005. 